\section{(9) OPEN ACCESS \\ Clinical, functional and radiographic consequences of achieving stable low disease activity and remission with adalimumab plus methotrexate or methotrexate alone in early rheumatoid arthritis: 26-week results from the randomised, controlled OPTIMA study}

\author{
Arthur Kavanaugh, ${ }^{1}$ Roy M Fleischmann, ${ }^{2}$ Paul Emery, ${ }^{3}$ Hartmut Kupper, ${ }^{4}$ Laura \\ Redden, ${ }^{5}$ Benoit Guerette, ${ }^{5}$ Sourav Santra, ${ }^{5}$ Josef S Smolen ${ }^{6}$
}

\begin{abstract}
- Additional figures are published online only. To view these files please visit the journal online (http:/dx.doi.org/ 10.1136/annrheumdis-2011201247).

1 Division of Rheumatology Allergy and Immunology, UCSD, San Diego, California, USA ${ }^{2}$ Division of Rheumatology, University of Texas Southwestern Medical Center at Dallas, Dallas, Texas, USA ${ }^{3}$ Section of Musculoskeletal Disease, University of Leeds, Leeds, UK

${ }^{4} \mathrm{GPRD}$, Abbott Laboratories, Ludwigshafen, Germany ${ }^{5} \mathrm{GPRD}$, Abbott Laboratories, Abbott Park, Illinois, USA ${ }^{6}$ Division of Rheumatology, Medical University of Vienna, Vienna, Austria
\end{abstract}

Correspondence to Arthur Kavanaugh, UCSD, UCSD, Rheumatology, Allergy, Immunology, 9500 Gilman Drive, MC 0943 La Jolla CA 92093-0943, USA: akavanaugh@ucsd.edu

Accepted 23 March 2012 Published Online First 19 May 2012

\begin{abstract}
Objective To assess the efficacy and safety of adalimumab plus methotrexate (ADA + MTX) compared with methotrexate monotherapy in achieving stable low disease activity (LDA; disease activity score (DAS28(CRP)) <3.2 at weeks 22 and 26) and clinical, radiographic and functional outcomes in methotrexatenaive patients with early rheumatoid arthritis (RA). Methods 1032 patients with active RA were randomly assigned 1:1 to ADA+MTX or placebo plus methotrexate (PBO + MTX) for 26 weeks. Treatment modifications were to be made in a subsequent study period based on the achievement of DAS28(CRP) $<3.2$ at weeks 22 and 26 . Post-hoc analyses compared patients achieving stable remission using DAS28 and 2010 ACR/EULAR criteria with those achieving LDA but not remission.

Results Among patients completing 6 months, 44\% (207/466) ADA + MTX versus 24\% (112/460) PBO+MTX patients achieved stable LDA at weeks 22 and 26 $(p<0.001)$. Combination therapy was statistically superior to methotrexate in obtaining higher ACR20/50/70 responses, more clinical remissions, greater mean reductions in DAS28(CRP), no radiographic progression, and normal functional status at week 26 ( $p<0.001$ for all). The only factor predicting stable LDA was disease activity at week 12. Patients achieving ACR/EULAR remission, particularly in the PBO+MTX group, had some advantage in radiographic outcomes compared with patients who only achieved LDA (but not remission). The overall frequency of adverse events was comparable between groups. There were more serious infections and deaths in the ADA+MTX group, with a possible age effect. Conclusions Treatment with ADA+MTX was significantly superior to methotrexate alone with respect to clinical, radiographic and functional outcomes in patients with early active RA. Before initiating treatment with adalimumab, individual patient evaluation of the benefit/risk ratio should be carefully considered.
\end{abstract}

The development of effective biological diseasemodifying antirheumatic drugs (DMARD), such as tumour necrosis factor $\alpha$ (TNF) inhibitors, especially in combination with methotrexate, has significantly improved treatment outcomes in rheumatoid arthritis (RA). Some patients with early RA may achieve significant benefit from methotrexate monotherapy, without the need for further therapy with biologic agents. However, it has not been consistently possible to identify such patients a priori.

With greater clinical success, it has been suggested that the management of RA should shift towards earlier, more intensive treatment strategies with sustained remission, or the lowest possible disease activity, as the ultimate goal of therapy. ${ }^{12}$ This is the essence of the 'treat-to-target' theory: abrogation of inflammation should prevent joint damage and preserve physical function, consequently improving overall quality of life. ${ }^{1}$ The focus on a goal of remission has resulted in the recent introduction of stringent American College of Rheumatology/European League against Rheumatism (ACR/EULAR) remission criteria. ${ }^{3}$ Potential differences in outcomes between patients achieving remission and those achieving low disease activity (LDA) have not been fully explored.

Optimal Protocol for Treatment Initiation with Methotrexate and Adalimumab (OPTIMA) was a phase 4, multinational, randomised, double-blind, controlled trial conducted in two periods. In the first period, reported here, patients with early active RA were randomly assigned to receive adalimumab plus methotrexate (ADA+MTX) or placebo plus methotrexate $(\mathrm{PBO}+\mathrm{MTX})$ for 26 weeks. Clinical, functional and radiographic outcomes were assessed, including indicators of response in each of the groups, and differences in outcomes between patients who achieved increasingly stringent treatment targets. In the second period, patients were followed for an additional year after potential treatment adjustments based on achieving a stable LDA target.

\section{PATIENTS AND METHODS}

\section{Patients}

Eligible patients were 18 years or older with a diagnosis of RA based on the 1987 ACR classification criteria, ${ }^{4}$ with a disease duration from diagnosis of less than 1 year. Patients must have met the following five criteria: (1) disease activity score based on 28 joints (DAS28) and C-reactive protein (CRP) greater than 3.2 ; (2) tender joint count based on 68 joints (TJC68) of 8 or greater; 
(3) swollen joint count based on 66 joints (SJC66) of 6 or greater; (4) erythrocyte sedimentation rate of $28 \mathrm{~mm} / \mathrm{h}$ or greater or $\mathrm{CRP}$ of $1.5 \mathrm{mg} / \mathrm{dl}$ or greater and (5) either rheumatoid factor (RF) positive, anticyclic citrullinated peptide positive, or more than one erosion on the hands or feet by $\mathrm{x}$-ray. Patients with a latent tuberculosis diagnosis based on positive purified protein derivative testing results according to local guidelines or other evidence were accepted if treatment for latent tuberculosis began before the administration of study drug. Patients who had received previous methotrexate, more than two synthetic DMARD, antiTNF therapy, or any other biological DMARD were excluded, as were those receiving intra-articular or parenteral corticosteroids in the 4 weeks preceding screening. All patients provided written informed consent.

\section{Study design}

OPTIMA (NCT00420927) was conducted at 161 centres in North and South America, Europe, Africa, New Zealand and Australia from December 2006 to July 2010. A central institutional review board or independent ethics committee approved the study at each site; the study was conducted in accordance with the International Conference on Harmonisation good clinical practices and the Declaration of Helsinki. Patients were centrally randomly assigned 1:1 using an interactive voice response system to adalimumab ( $40 \mathrm{mg}$ every other week) plus methotrexate (titrated to $20 \mathrm{mg}$ /week by week 8 ) or PBO+MTX for 26 weeks (figure 1).

\section{Effi cacy outcomes}

The primary endpoint of the study was a composite of DAS28(CRP) less than 3.2 at week 78 and no radiographic

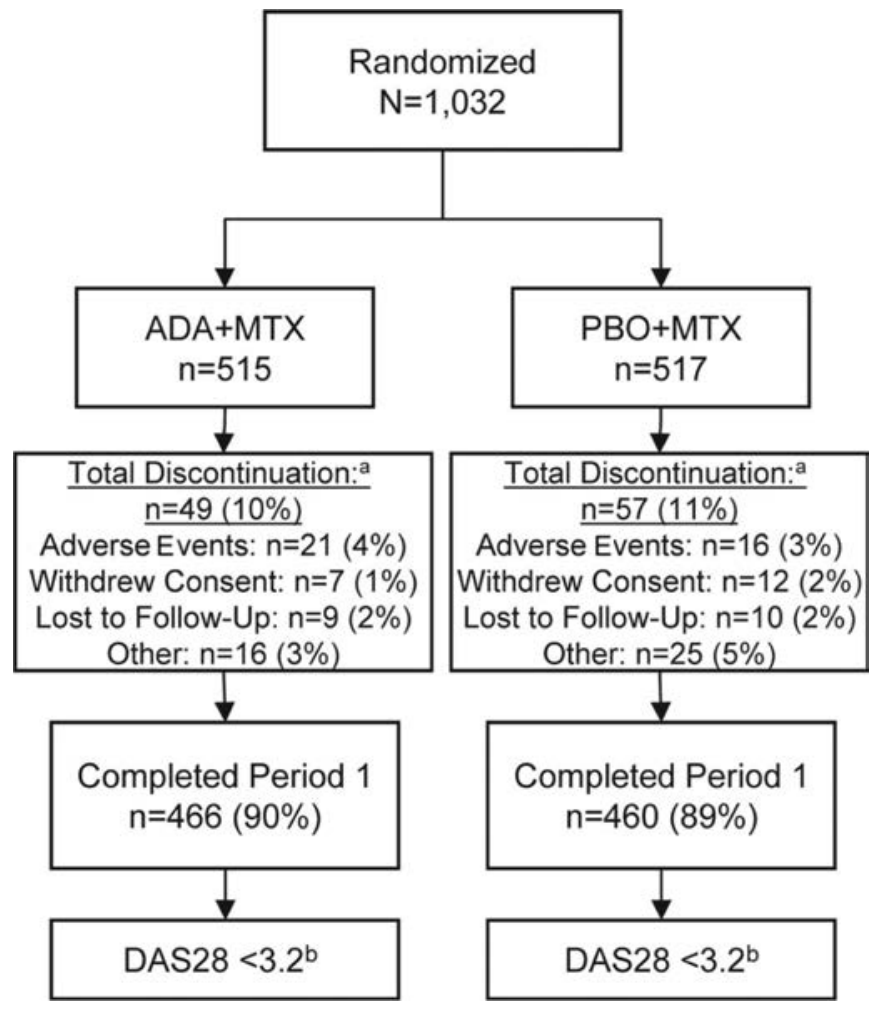

Figure 1 Study design and patients' disposition to 26 weeks. anvestigators may have listed more than one reason. bStable low disease activity target defined as disease activity score in 28 joints (DAS28) C-reactive protein less than 3.2 at weeks 22 and 26. ADA, adalimumab; MTX, methotrexate; PBO, placebo. progression from baseline to week 78 in the van der Heijde modified total Sharp score $(\triangle \mathrm{mTSS} \leq 0.5)$. There were multiple secondary endpoints including ACR20/50/70 response, DAS28 less than 3.2, DAS28 less than 2.6, simplified disease activity index (SDAI) of 11 or less, SDAI of 3.3 or less, which were also planned for week 78. In this report, the percentage of patients achieving a stable LDA target of DAS28(CRP) less than 3.2 at weeks 22 and 26 was compared between treatment groups. Clinical assessments during period 1 were performed at baseline and weeks 2, 4, 8, 12, 22 and 26. Clinical improvement was further assessed using ACR 20/50/70, ${ }^{5} \mathrm{DAS} 28(\mathrm{CRP})^{6}$ and the SDAI, ${ }^{7}$ including the index-based definition of the 2010 ACR/EULAR provisional remission criteria, SDAI of 3.3 or less. ${ }^{3}$ Reductions in these individual measures were also assessed: tender and swollen joint counts, patient's and physician's global assessment of disease activity, serum CRP levels and the health assessment questionnaire disability index (HAQ-DI). Normal physical function was defined as HAQ-DI less than 0.5. ${ }^{8}$ Radiographs of the hands/wrists and feet were obtained at baseline and week 26 , and mean changes in the mTSS, joint erosion and joint space narrowing were used to evaluate radiographic progression; ${ }^{9}$ no radiographic progression was defined as $\triangle \mathrm{mTSS}$ of 0.5 or less.

\section{Safety}

Treatment-emergent adverse events (AE) were defined as events with an onset date that was on or after the first dose of study drug, and up to 70 days after the last dose for patients who discontinued prematurely.

\section{Statistical analysis}

The intent-to-treat population included all randomly assigned patients who received at least one dose of study drug. For categorical clinical and functional outcomes, a non-responder imputation approach was used, such that patients with missing responses were considered non-responders. The percentage of patients achieving the protocol-specified stable LDA target was evaluated among week 26 completers. Last observation carried forward analyses were used for continuous clinical and functional outcomes. Multiple imputation ${ }^{10}$ was used to assess radiographic data. Using the Markov chain Monte Carlo method, missing radiographic data were imputed 10 times. AE were reported as the number and percentages of patients with $\mathrm{AE}$ by treatment group.

Categorical efficacy variables were analysed using Pearson's $\chi^{2}$ test or Fisher's exact test; evaluations of continuous variables were based on an analysis of covariance model adjusting for baseline. All statistical tests were two sided, and $p<0.05$ was considered statistically significant. $p$ Values for continuous variables were based on between-group mean changes.

Regression analyses examined variables associated with the stable LDA target (DAS28(CRP) $<3.2$ at both weeks 22 and 26) and with stable remission (SDAI $\leq 3.3$ at both weeks 22 and 26). Continuous variables included CRP, SJC66 and patient's and physician's global assessments of disease activity at baseline and week 12. Categorical variables included sex, smoking status, concomitant steroid use at baseline, joint erosions at baseline, erosions in the metatarsophalangeal joints at baseline, copy number of HLA-DRB1 shared epitope $(0 \times / 1 \times / 2 \times)$, IL-4R allele (AA/AG/ GG) and FcrRIIb allele (TT/TC/CC), week 12 responder for DAS28(CRP) improvement (decrease $>0.6 />1.2 />1.8$ ) and week 12 responders for EULAR good and moderate improvement. Variables with significant effects $(p<0.10)$ in univariate analysis were selected for step-wise selection in multivariate regression. 
Post-hoc analyses evaluated outcomes within treatment groups for patients who achieved DAS28(CRP) less than 3.2 but not SDAI of 3.3 or less at both weeks 22 and 26 with patients who achieved stable SDAI of 3.3 or less at both weeks 22 and 26; similar analyses were done for patients with DAS28 less than 2.6 but not SDAI of 3.3 or less. Observed completer analyses were used for these outcomes.

\section{RESULTS}

\section{Patient disposition}

A total of 1032 patients was randomly assigned to receive $\mathrm{ADA}+\mathrm{MTX}(\mathrm{n}=515)$ or PBO+MTX ( $\mathrm{n}=517$; figure 1$)$. Nine hundred and twenty-six (90\%) patients completed 26 weeks, with a comparable proportion and similar reasons for discontinuation between groups.

\section{Baseline demographics and disease characteristics}

Patient demographics and disease characteristics were comparable between groups and were representative of patients with early, active RA (table 1). The mean duration of RA from diagnosis was approximately 4 months. As expected based

Table 1 Demographics and disease characteristics at baseline (intent-to-treat population)

\begin{tabular}{|c|c|c|}
\hline Characteristics* & $\begin{array}{l}\text { ADA+MTX } \\
(n=515)\end{array}$ & $\begin{array}{l}\text { PBO+MTX } \\
(\mathrm{n}=517)\end{array}$ \\
\hline \multicolumn{3}{|l|}{ Demographics } \\
\hline Female, $\mathrm{n}(\%)$ & $380(74)$ & $382(74)$ \\
\hline White, $\mathrm{n}(\%)$ & $460(89)$ & $464(90)$ \\
\hline Age, mean (SD), years & $50.7(14.5)$ & $50.4(13.6)$ \\
\hline Disease duration, mean (SD), months & $4.0(3.6)$ & $4.5(7.2)$ \\
\hline Previous DMARD use, $\mathrm{n}(\%)$ & $54(11)$ & $51(10)$ \\
\hline Concomitant systemic corticosteroid use, $\mathrm{n}(\%)$ & $212(41)$ & $239(46)$ \\
\hline Concomitant NSAID use, $\mathrm{n}(\%)$ & $401(78)$ & $410(79)$ \\
\hline \multicolumn{3}{|l|}{ Disease characteristics } \\
\hline $\mathrm{RF}+, \mathrm{n}(\%) \dagger$ & $445(87)$ & $457(89)$ \\
\hline Anti-CCP,$+ \mathrm{n}(\%) \ddagger$ & $426(84)$ & $430(84)$ \\
\hline CRP, mean (SD), mg/l & $27(32)$ & $30(33)$ \\
\hline TJC (0-28; 0-68), mean (SD) & $16(6.6) ; 29(15)$ & $16(6.7) ; 27(15)$ \\
\hline SJC (0-28; 0-66), mean (SD) & $13(5.8) ; 18(11)$ & 12 (5.8); 18 (11 \\
\hline DAS28(CRP), mean (SD)§ & $6.0(1.0)$ & $6.0(1.0)$ \\
\hline DAS28(CRP) $\geq 5.1, n(\%) \S$ & $421(83)$ & $403(80)$ \\
\hline SDAI, mean (SD) & $44.0(14.2)$ & $43.3(14.6)$ \\
\hline SDAI > 26, n (\%) & $459(89)$ & $453(88)$ \\
\hline $\begin{array}{l}\text { Patient's pain assessment, mean (SD), 0-100 } \\
\text { mm VAS }\end{array}$ & $65(21)$ & $65(21)$ \\
\hline $\begin{array}{l}\text { Patient's global assessment, mean (SD), 0-100 } \\
\text { mm VAS }\end{array}$ & $64(23)$ & $63(22)$ \\
\hline $\begin{array}{l}\text { Physician's global assessment, mean (SD), } \\
0-100 \mathrm{~mm} \text { VAS** }\end{array}$ & $63(18)$ & $62(18)$ \\
\hline HAQ-DI (0-3), mean (SD)†† & $1.61(0.69)$ & $1.60(0.65)$ \\
\hline 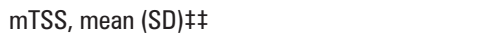 & $11.8(18.1)$ & $11.2(17.4)$ \\
\hline 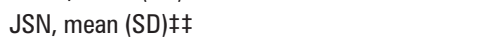 & $6.4(10.4)$ & $6.1(10.2)$ \\
\hline Joint erosion, mean (SD)‡‡ & $5.4(9.1)$ & $5.1(8.4)$ \\
\hline More than one erosion, $\mathrm{n}(\%) \ddagger \ddagger$ & $419(82)$ & $433(84)$ \\
\hline
\end{tabular}

${ }^{*} \mathrm{p}>0.05$ for all characteristics.

$\mathrm{tn}=510(\mathrm{ADA}+\mathrm{MTX})$ and $\mathrm{n}=511(\mathrm{PBO}+\mathrm{MTX})$

$\ddagger n=509(A D A+M T X)$ and $n=513(P B O+M T X)$

$\S n=507(A D A+M T X)$ and $n=505(P B O+M T X)$

In $=504($ ADA + MTX) and $n=505($ PBO + MTX)

${ }^{* *} \mathrm{n}=512($ ADA + MTX).

$+\dagger n=514(\mathrm{ADA}+\mathrm{MTX})$ and $\mathrm{n}=516(\mathrm{PBO}+\mathrm{MTX})$.

$\ddagger \ddagger n=508(A D A+M T X)$ and $n=514(P B O+M T X)$.

ADA, adalimumab; anti-CCP, anticyclic citrullinated peptide; CRP, $C$-reactive protein;

DAS28, disease activity score in 28 joints; DMARD, disease-modifying antirheumatic

drug; HAO-DI, health assessment questionnaire disability index; JSN, joint space

narrowing; MTX, methotrexate; NSAID, non-steroidal anti-inflammatory drug; PBO,

placebo; RF, rheumatoid factor; SD, standard deviation; SDAI, simplified disease activity

index; SJC, swollen joint count; TJC, tender joint count; mTSS, modified total Sharp

score; VAS, visual analogue scale. on inclusion criteria, the study population exhibited multiple poor prognostic factors including RF and/or anticyclic citrullinated peptide positivity, elevated CRP, a high number of swollen and tender joints, high disease activity by composite measures and joint damage. Previous and concomitant use of DMARD, non-steroidal anti-inflammatory drugs and systemic corticosteroids was comparable between treatment groups.

\section{Clinical and functional outcomes}

Among patients treated with ADA+MTX who completed 26 weeks and were eligible for re-randomisation, 44\% (207/466) achieved the stable LDA target, compared with 24\% (112/460) in the methotrexate monotherapy group $(\mathrm{p}<0.001$; figure $2 \mathrm{~A})$. There were $52 \%(240 / 466)$ and $34 \%(155 / 460)$ of patients with LDA at week 22, and 51\% (238/466) and 29\% (132/460) with LDA at week 26 among ADA+MTX and PBO+MTX patients, respectively, who completed 26 weeks.

Significantly more patients treated with ADA+MTX also demonstrated clinical improvements according to ACR20/50/70 response rates compared with methotrexate monotherapy at week 26 (figure $2 \mathrm{~B}$ ). Patients treated with ADA+MTX had more rapid clinical improvements, detectable as early as week 2 ( $\mathrm{p}<0.001$; supplementary figure S1, available online only) and a greater and more rapid decline in mean DAS28(CRP) to 26 weeks compared with those treated with methotrexate monotherapy $(p<0.001$ weeks 2-26; supplementary figure S2A, available online only); mean DAS28(CRP) values at week 26 were 3.3 in the ADA+MTX group and 4.1 in the PBO+MTX group. Significant improvements in each of the core components of DAS28(CRP) and other individual measures were observed at 2 weeks and maintained to 26 weeks in patients treated with ADA+MTX versus methotrexate monotherapy ( $<<0.001$; supplementary figure S2B-F, available online only). Approximately twice as many patients in the ADA+MTX group achieved remission or LDA compared with methotrexate monotherapy ( $p<0.001$; figure $2 \mathrm{C}$ ). In addition to improving clinical signs and symptoms, treatment with $\mathrm{ADA}+\mathrm{MTX}$ resulted in rapid and significantly greater mean functional improvements compared with methotrexate monotherapy ( $p<0.001$; figure $2 \mathrm{D})$. At week 26 , a greater proportion of combination therapy-treated patients demonstrated normal function, compared with those treated with $\mathrm{PBO}+\mathrm{MTX}$ ( $40 \%$ vs $28 \%$, respectively; $\mathrm{p}<0.001$ ).

\section{Radiographic outcomes}

Patients treated with ADA+MTX had significantly less radiographic progression compared with those receiving $\mathrm{PBO}+\mathrm{MTX}$ $(p<0.001$; figure 3A). Probability plots show that the difference between groups was driven by a subset of patients treated with methotrexate monotherapy who had greater progression than those treated with ADA+MTX (figure 3B). After 26 weeks, $87 \%$ of patients receiving combination therapy had no evidence of radiographic progression, compared with $72 \%$ of patients in the $\mathrm{PBO}+\mathrm{MTX}$ group $(\mathrm{p}<0.001)$.

\section{Achieving stable LDA and remission}

Predictors of stable LDA and remission

Generally, lower scores for the DAS28(CRP) components, at both baseline and week 12 , were significantly associated with achieving DAS28(CRP) LDA at weeks 22 and 26 by univariate analysis, as were meeting DAS28-based response criteria at week 12 (ie, EULAR good response). Following multivariate 
a

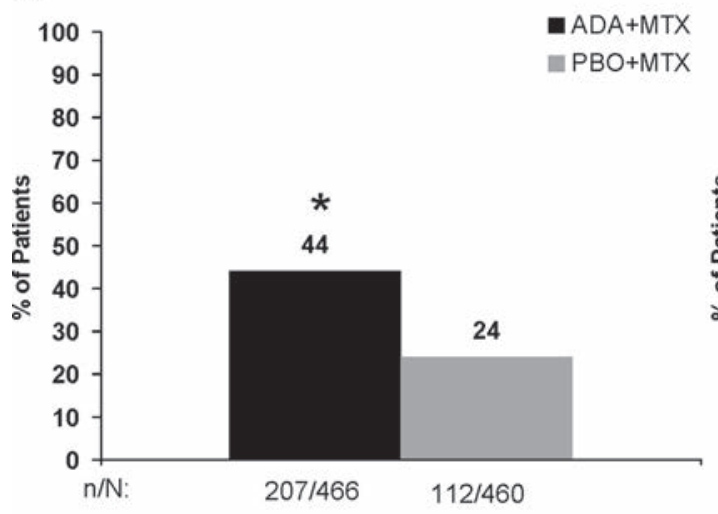

.

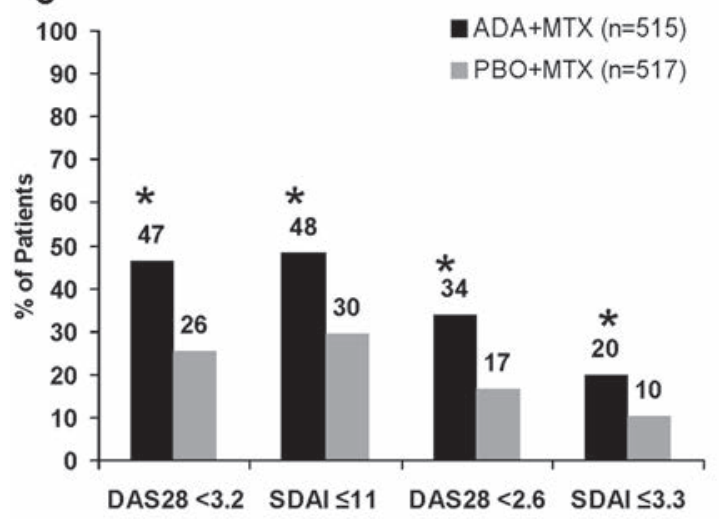

b

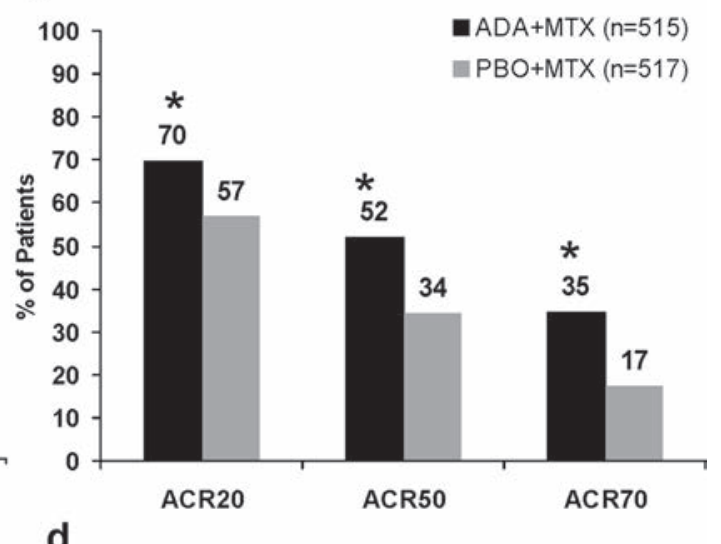

d

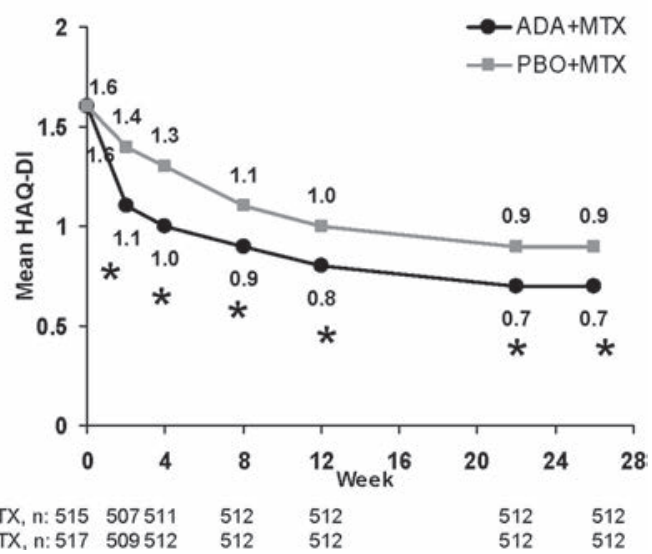

Figure 2 Clinical and functional improvements with adalimumab plus methotrexate (ADA+MTX) or placebo plus methotrexate (PBO+MTX) to 26 weeks (A). Percentage of patients achieving the stable low disease activity target (disease activity score in 28 joints (DAS28) C-reactive protein $<3.2$ ) at weeks 22 and 26 among patients who completed week 26 (B). Percentage of patients achieving American College of Rheumatology (ACR) 20/50/70 responses at week 26 (C). Percentage of patients achieving low disease activity and remission at week 26 (D). Mean health assessment questionnaire disability index (HAO-DI) values to week 26 . ${ }^{*} \mathrm{p}<0.001$ versus PBO+MTX by $\chi^{2}$ test, non-responder imputation analysis for completers (A) or intent-to-treat $(B, C)$; by analysis of covariance, last observation carried forward analysis (D). SDAl, simplified disease activity index.

selection, lower disease activity levels at week 12 were predictive of higher chances of achieving the stable LDA target for both treatment strategies. Decreased numbers of swollen joints and lower patient's global assessment scores at week 12 were modestly associated with reaching the stable LDA target for both ADA+MTX (p values: SJC66 OR 1.14 (95\% CI 1.08 to 1.20 ), $p<0.001$; patient's global OR 1.05 (95\% CI 1.04 to 1.07), $\mathrm{p}<0.001$ ) and for methotrexate monotherapy (SJC66 OR 1.19 (95\% CI 1.11 to 1.27$), \mathrm{p}<0.001$; patient's global OR 1.04 (95\% CI 1.02 to 1.05$), \mathrm{p}<0.001)$. In addition, lower week 12 CRP levels were modestly associated with the ability to achieve DAS28(CRP) less than 3.2 at weeks 22 and 26 in the ADA+MTX group (OR 1.06; 95\% CI 1.04 to 1.07, $\mathrm{p}=0.03$ ). Low physician's global assessment at baseline was the only other predictive factor for achieving stable LDA with methotrexate monotherapy (OR 1.02; 95\% CI 1.00 to $1.03, \mathrm{p}=0.04$ ). Results of analyses evaluating factors associated with achieving SDAI of 3.3 or less at both weeks 22 and 26 were comparable with those for the stable LDA target. All other clinical and disease characteristics and putative biomarkers tested were not predictive of response in either group, including a categorical variable for concomitant steroid use, which was non-significant in univariate analysis for both treatment groups.
The consequences of achieving increasingly stringent stable therapeutic targets: DAS28(CRP) LDA/DAS28(CRP) remission/ACR/ EULAR remission.

Of patients who achieved the stable LDA target, $30 \%$ and $31 \%$ were also in stable SDAI remission in the ADA+MTX and $\mathrm{PBO}+\mathrm{MTX}$ groups, respectively (figure 4A).

Although the numbers became smaller in these subgroups, it consistently appeared that the radiographic benefits were proportionally greater in the $\mathrm{PBO}+\mathrm{MTX}$ group than in the $\mathrm{ADA}+\mathrm{MTX}$ group when more stringent targets were used. A greater percentage of patients who achieved stable SDAI remission had no radiographic progression, compared with those who only achieved stable DAS28(CRP) LDA, regardless of treatment (figure 4B). Patients who attained DAS28(CRP) LDA response alone had higher mTSS scores at week 26 while patients in SDAI remission exhibited no progression, but the differences were greater and achieved statistical significance only in the $\mathrm{PBO}+\mathrm{MTX}$ group (figure $4 \mathrm{C}$ ).

A greater proportion of methotrexate-treated patients who attained SDAI remission achieved normal function, compared with those in stable DAS28(CRP) LDA ( $p=0.004$; figure 4D). At week 26, patients in either treatment arm who achieved stable DAS28(CRP) LDA, but not SDAI remission had small, but significantly greater reductions in mean HAQ-DI compared with those 

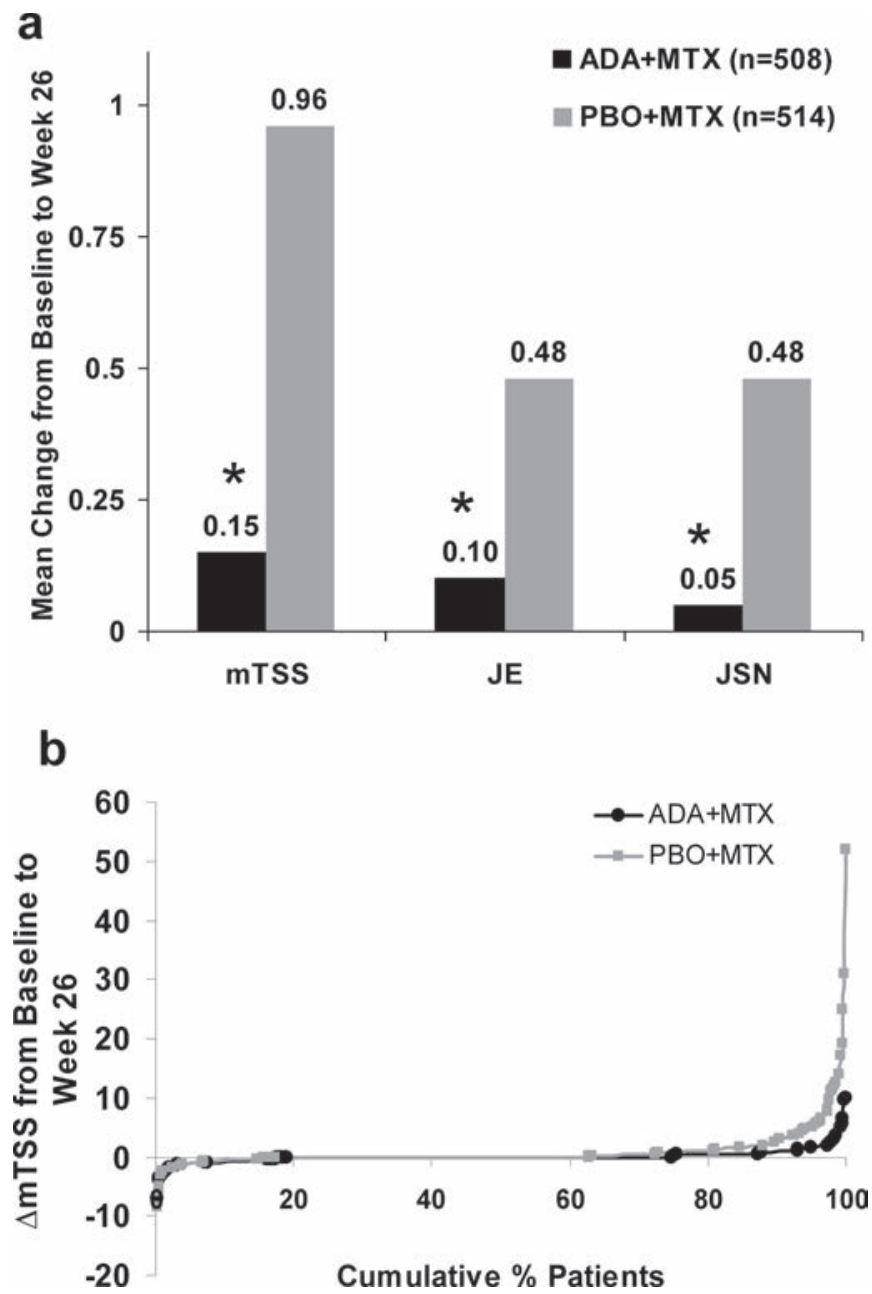

Figure 3 Radiographic changes with adalimumab plus methotrexate (ADA+MTX) or placebo plus methotrexate (PBO+MTX) from baseline to week 26 (A). Mean change in the van der Heijde modified total Sharp score (mTSS), joint erosion (JE) and joint space narrowing (JSN). ${ }^{*} p<0.001$ versus $\mathrm{PBO}+\mathrm{MTX}$ by analysis of covariance, multiple imputation analysis. (B). Cumulative probability plot of change in mTSS.

who attained stable SDAI remission ( $\mathrm{p}<0.05$ for both; figure 4E); however, the differences did not approach 0.22 , the threshold for minimally clinically important differences. Furthermore, the mean $\mathrm{HAQ}$ values were numerically lower in the SDAI remission group at baseline.

\section{Safety}

The frequencies of total $\mathrm{AE}$ and serious $\mathrm{AE}$ were similar between the ADA+MTX and PBO+MTX groups (table 2). The most frequently occurring $(>5 \%) \mathrm{AE}$ were nausea, upper respiratory tract infection and nasopharyngitis in the ADA+MTX group, and nausea, upper respiratory tract infection and diarrhoea in the $\mathrm{PBO}+\mathrm{MTX}$ group. A similar percentage of patients in both treatment groups reported infections $(37.1 \%$ and $36.4 \%$ in the $\mathrm{ADA}+\mathrm{MTX}$ and $\mathrm{PBO}+\mathrm{MTX}$ groups, respectively). Serious infections occurred at a higher frequency in the ADA+MTX group versus methotrexate monotherapy $(13(2.5 \%)$ vs $6(1.2 \%)$, respectively). Pneumocystis jiroveci pneumonia, an opportunistic infection, occurred in one patient in the ADA+MTX group; three patients in the $\mathrm{PBO}+\mathrm{MTX}$ groups also had opportunistic infections, namely candidiasis, oral candidiasis and gastrointestinal candidiasis. None were serious, and all resolved. Two malignancies (malignant melanoma in situ and squamous cell carcinoma) were reported in the ADA+MTX group. There were no cases of lymphoma or demyelinating disease; one case of lupus-like syndrome was reported in the ADA+MTX group.

Seven deaths were reported: six in the ADA+MTX group and one in the PBO+MTX group. A 36-year-old man in the $\mathrm{PBO}+\mathrm{MTX}$ group experienced sudden death; this patient had a history of type 1 diabetes, hyperlipidaemia, hypertension and smoking. Among patients treated with ADA+MTX, a 45-yearold woman died from septic shock following peritoneal tuberculosis and a 61-year-old man experienced right ventricular failure following bronchopneumonia (positive for Mycobacterium abscessus and Candida kefyr). Interestingly, the remaining four deaths in the ADA+MTX group were in patients older than 70 years: a 74-year-old man with a history of cardiomyopathy, hyperlipidaemia and smoking who died from unknown cause preceded in close proximity by pharyngitis; a 73-year-old woman with a history of chronic obstructive pulmonary disease, smoking and an abnormal baseline chest x-ray who had acute respiratory distress syndrome preceded by pneumonia; a 74-year-old man with pleural scarring, a history of smoking and abnormal baseline chest $\mathrm{x}$-ray whose death was attributed to interstitial lung disease; and an 83-year-old woman with pleural scarring, a history of smoking and abnormal baseline chest x-ray whose death was also attributed to interstitial lung disease, with positive culture for Klebsiella pneumoniae. 

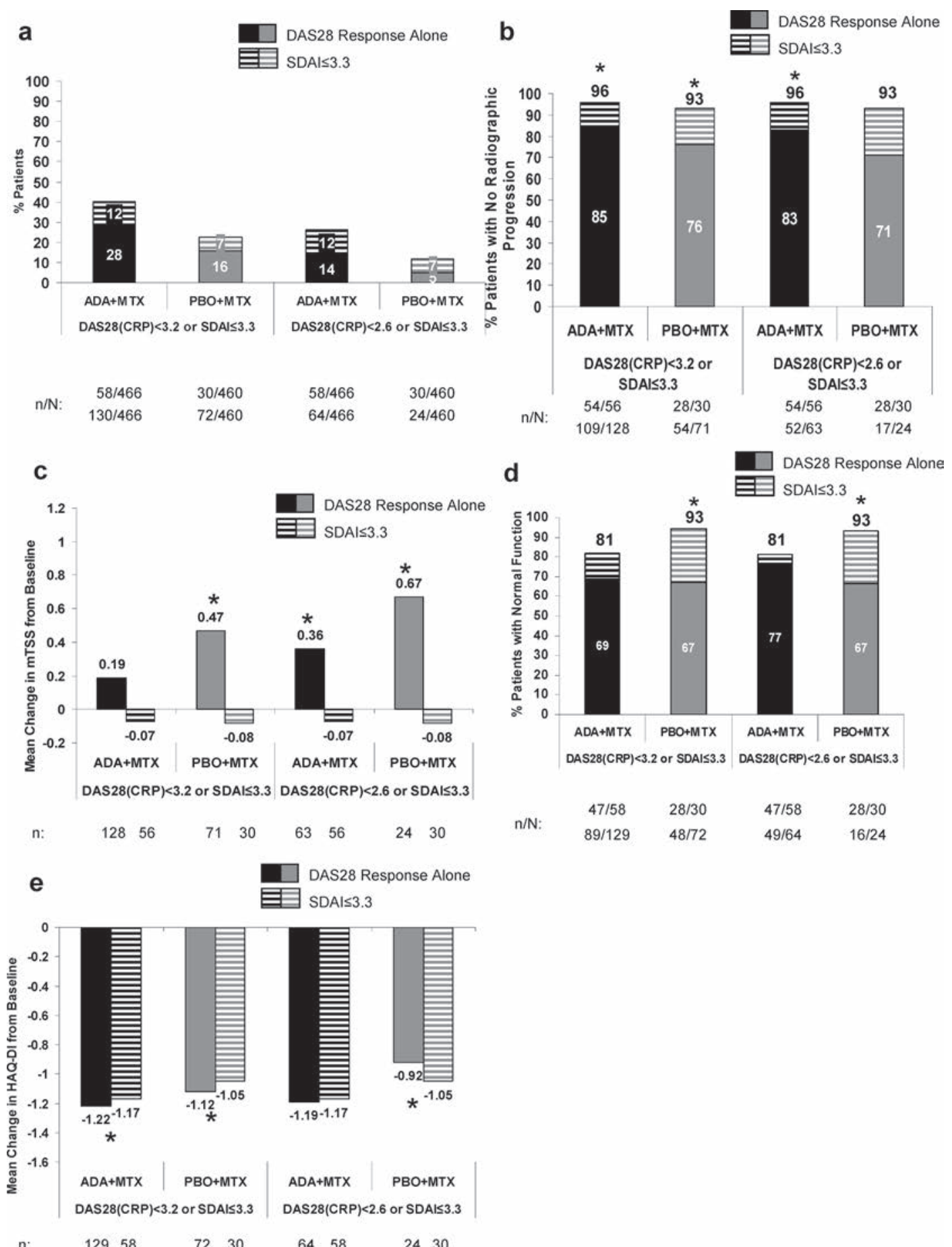

Figure 4 Radiographic and functional consequences of achieving increasingly stringent targets (stable disease activity score in 28 joints (DAS28) C-reactive protein (CRP) low disease activity or remission vs simplified disease activity index (SDAl) remission) (A). Percentage of patients exclusively achieving stable DAS28(CRP) targets versus SDAl remission at weeks 22 and 26 (B). Percentage of patients with no radiographic progression (van der Heijde modified total Sharp score $(\triangle \mathrm{mTSS}) \leq 0.5)$ at week 26 among those who exclusively achieved stable DAS28(CRP) targets or SDAI remission at weeks 22 and 26 (C). Mean $\triangle$ mTSS from baseline to week 26 among patients who exclusively achieved stable DAS28(CRP) targets or SDAI remission (D). Percentage of patients with normal function among those who exclusively achieved stable DAS28(CRP) targets or SDAI remission at weeks 22 and 26 (E). Mean change in health assessment questionnaire disability index (HAO-DI) at week 26 in patients in who exclusively achieved stable DAS28(CRP) targets or SDAl remission at weeks 22 and $26 .{ }^{*} \mathrm{p}<0.05$ for DAS28(CRP) response versus SDAl of 3.3 or less within treatment group by $\chi^{2}$ test, observed analysis among week 26 completers $(B, D)$ or by analysis of covariance, observed analysis $(C, E)$. ADA+MTX, adalimumab plus methotrexate; PBO+MTX, placebo plus methotrexate. 
Table 2 Number and percentage of AE of interest by treatment group

\begin{tabular}{lcc}
\hline AE, $\mathbf{n}(\%)$ patients & $\begin{array}{c}\text { ADA+MTX } \\
(\mathbf{n = 5 1 5 )}\end{array}$ & $\begin{array}{c}\text { PBO+MTX } \\
(\mathbf{n}=517)\end{array}$ \\
\hline Any AE & $379(73.6)$ & $368(71.2)$ \\
AE leading to discontinuation of study drug & $26(5.0)$ & $15(2.9)$ \\
Serious AE & $37(7.2)$ & $32(6.2)$ \\
Serious infectious AE & $13(2.5)$ & $6(1.2)$ \\
Opportunistic infections (excluding tuberculosis) & $1(0.2)$ & $3(0.6)$ \\
Tuberculosis & $1(0.2)$ & 0 \\
Malignancies (excluding NMSC) & $1(0.2)$ & 0 \\
NMSC & $1(0.2)$ & 0 \\
Congestive heart failure related & $1(0.2)$ & $1(0.2)$ \\
Fatal AE & $6(1.2)^{*}$ & $1(0.2)$ \\
\hline
\end{tabular}

*Includes one non-treatment-emergent death.

ADA, adalimumab; AE, adverse events; MTX, methotrexate; NMSC, non-melanoma skin cancer; $\mathrm{PBO}$, placebo

\section{DISCUSSION}

This first period of OPTIMA allowed not only an assessment of the proportion of early RA patients who achieved the main current treatment targets, but also evaluated the stringent new ACR/EULAR remission criteria, and the impact of achieving various therapeutic targets on radiographic and functional outcomes.

These results showed that starting treatment with ADA+MTX resulted in rapid clinical and functional improvements that were maintained to 26 weeks. At 26 weeks, approximately twice as many ADA+MTX-treated patients attained states of LDA and remission, including the new 2010 provisional ACR/EULAR remission criteria and the stable LDA target of DAS28(CRP) less than 3.2 at weeks 22 and 26, compared with PBO+MTX-treated patients, with significant clinical and functional differences observed as early as week 2 .

OPTIMA extends the findings of previous trials of TNF inhibitors in early RA, including PREMIER. ${ }^{11}$ Clinical outcomes observed at week 26 in OPTIMA were comparable to those at week 52 in PREMIER. However, the mean disease duration in OPTIMA was 4 months and thus the shortest among multinational clinical trials of TNF inhibitors in early RA. As an especially aggressive patient cohort was recruited, not surprisingly, patients exhibited the presence of poor prognostic markers, such as elevated CRP, high swollen joint counts, existing joint damage and a high frequency $(>80 \%)$ of autoantibodies (RF and/or anticitrullinated protein antibodies). Given the risk of joint damage potentially associated with these factors, significant radiographic progression was already seen at 26 weeks in the methotrexate monotherapy group, while significant inhibition of joint damage was observed with ADA+MTX treatment, supporting the recommendation for the aggressive treatment of patients with early, active RA and poor prognostic factors, particularly if patients do not attain LDA on methotrexate. ${ }^{2}$

It is of great interest and utility to clinicians to predict clinical outcomes a priori or even early into a course of therapy. In this study, the only predictors of clinical outcome at week 26 were measures of ongoing disease activity after 12 weeks of treatment, which is in alignment with treatment guidelines. This is a logical result, given that these factors are components of the score used to assess LDA. These data confirm that responses within 3 months of treatment initiation are predictive of lower disease activity long term. ${ }^{12-14}$

For patients who respond to treatment, the radiographic and functional consequences of achieving LDA versus remission are largely unexplored. When fulfilling ACR/EULAR index-based remission criteria, there was no mean progression of joint damage in either treatment arm. Not surprisingly, methotrexatetreated patients experienced significantly higher progression of joint damage when less stringent outcomes were used, such as DAS28(CRP) remission or LDA, compared with the new ACR/EULAR criteria. In contrast, radiographic benefits were comparable and robust in ADA+MTX-treated patients, irrespective of the achievement of stable outcomes, consistent with previous observations. ${ }^{15}$ Nevertheless, as radiographic progression is very low even in LDA, ${ }^{16}$ our results suggest that an LDA target is reasonable and desirable for many patients. Similar findings were observed when assessing functional benefits for either treatment arm, namely lower $\mathrm{HAQ}$ values with increasing stringency of criteria. In sum, despite the smaller numbers of patients in the post-hoc subgroup analysis, it appears that both the stringency of the target and the treatment utilised impacted radiographic and functional outcomes.

Most AE in this study were comparable to those observed in other studies of TNF inhibitors. ${ }^{17} 18$ The overall frequency of serious infections was consistent with the known safety profile for adalimumab. More deaths were observed in ADA+MTX patients versus $\mathrm{PBO}+\mathrm{MTX}$ patients, which is inconsistent with previous clinical trial experience. ${ }^{18}$ Advanced age and comorbidities have been reported in some analyses as risk factors for immunomodulatory therapy toxicity. ${ }^{19-22}$ Older patients were over-represented, with four of six deaths in the ADA+MTX arm occurring in patients over 70 years old. In retrospect, the prevalence of pre-existing comorbidities in these patients appeared as possible contributing factors. Although adalimumab conferred enhanced clinical, functional and radiographic outcomes, the risks of treatment should be considered before initiating therapy, particularly in older patients and those with comorbidities or conditions that may predispose them to infection.

In summary, significant improvements in clinical and functional outcomes were observed as early as week 2 and continued to week 26 with ADA+MTX treatment. Combination therapy allowed one in five patients to achieve stringent remission according to the new ACR/EULAR definition within 6 months of treatment initiation, and an additional almost $30 \%$ achieved LDA; the data also reveal that approximately half of these proportions can be attained with methotrexate monotherapy. Early intervention with combination therapy also offered substantial protection from radiographic damage compared with methotrexate monotherapy. These results expand information on the use of combination methotrexate plus TNF inhibitors as a treatment option with substantial therapeutic benefits in patients with early, active RA. As with the use of any potent immunomodulatory therapy, patients should be appropriately screened before and closely monitored during treatment for relevant safety concerns.

Contributors Abbott Laboratories sponsored this study (NCT00420927), led development of the study design in collaboration with academic investigators, and analysed the primary data. All authors contributed to design, analysis, and interpretation of these data, and reviewed, approved, and decided to publish the manuscript.

Acknowledgements Elaine M Smith and Theresa J Peterson, of Abbott, drafted and revised the manuscript.

Funding This study was funded by Abbott Laboratories.

Competing interests AK has provided expert advice to, and/or received research grants from Abbott, Amgen, Astra-Zeneca, BMS, Celgene, Centocor-Janssen, Pfizer, Roche and UCB. RMF has received research grants and consulting fees or other remuneration from Abbott. PE has provided expert advice and undertaken trials for Abbott, Merck, Pfizer, UCB, Roche and BMS. HK, LR, BG and SS are Abbott employees and may hold stock or options. JSS has received research grants and/ or consulting fees or other remuneration from Abbott, Amgen, Astra-Zeneca, BMS, 
Celgene Centocor-Janssen, Glaxo, Lilly, Pfizer (Wyeth), MSD (Schering-Plough), Novo-Nordisk, Roche, Sandoz and UCB.

Ethics approval A central institutional review board or independent ethics committee approved the study at each site.

Patient consent Obtained.

Provenance and peer review Not commissioned; externally peer reviewed.

Open Access This is an Open Access article distributed in accordance with the Creative Commons Attribution Non Commercial (CC BY-NC 3.0) license, which permits others to distribute, remix, adapt, build upon this work non-commercially, and license their derivative works on different terms, provided the original work is properly cited and the use is non-commercial. See: http://creativecommons.org/ licenses/by-nc/3.0/

\section{REFERENCES}

1. Smolen JS, Aletaha D, Bijlsma JW, et al. Treating rheumatoid arthritis to target: recommendations of an international task force. Ann Rheum Dis 2010;69:631-7.

2. Saag KG, Teng GG, Patkar NM, et al. American College of Rheumatology 2008 recommendations for the use of nonbiologic and biologic disease-modifying antirheumatic drugs in rheumatoid arthritis. Arthritis Rheum 2008;59:762-84.

3. Felson DT, Smolen JS, Wells G, et al. American College of Rheumatology/European League against Rheumatism provisional definition of remission in rheumatoid arthritis for clinical trials. Ann Rheum Dis 2011;70:404-13.

4. Arnett FC, Edworthy SM, Bloch DA, et al. The American Rheumatism Association 1987 revised criteria for the classification of rheumatoid arthritis. Arthritis Rheum 1988;31:315-24.

5. Felson DT, Anderson JJ, Boers M, et al. American College of Rheumatology Preliminary definition of improvement in rheumatoid arthritis. Arthritis Rheum 1995;38:727-35.

6. Prevoo ML, van 't Hof MA, Kuper HH, et al. Modified disease activity scores that include twenty-eight-joint counts. Development and validation in a prospective longitudinal study of patients with rheumatoid arthritis. Arthritis Rheum 1995;38:44-8.

7. Smolen JS, Breedveld FC, Schiff MH, et al. A simplified disease activity index for rheumatoid arthritis for use in clinical practice. Rheumatology (Oxford) 2003:42:244-57.

8. Fries JF, Spitz P, Kraines RG, et al. Measurement of patient outcome in arthritis. Arthritis Rheum 1980;23:137-45.

9. van der Heijde D, How to read radiographs according to the Sharp/van der Heijde method.J Rheumato/2000;27:261-3.

10. Baron G, Ravaud P, Samson A, et al. Missing data in randomized controlled trials of rheumatoid arthritis with radiographic outcomes: a simulation study. Arthritis Rheum 2008:59:25-31.
11. Breedveld FC, Weisman MH, Kavanaugh AF, et al. The PREMIER study: A multicenter, randomized, double-blind clinical trial of combination therapy with adalimumab plus methotrexate versus methotrexate alone or adalimumab alone in patients with early, aggressive rheumatoid arthritis who had not had previous methotrexate treatment. Arthritis Rheum 2006:54:26-37.

12. Aletaha D, Funovits J, Keystone EC, et al. Disease activity early in the course of treatment predicts response to therapy after one year in rheumatoid arthritis patients. Arthritis Rheum 2007;56:3226-35

13. Curtis JR, Yang S, Chen L, et al. Predicting low disease activity and remission using early treatment response to antitumour necrosis factor therapy in patients with rheumatoid arthritis: exploratory analyses from the TEMPO trial. Ann Rheum Dis 2012:71:206-12.

14. Bakker MF, Jacobs JW, Welsing PM, et al. Early clinical response to treatment predicts 5-year outcome in RA patients: follow-up results from the CAMERA study. Ann Rheum Dis 2011;70:1099-103.

15. Emery $\mathbf{P}$, Genovese $\mathrm{MC}$, van Vollenhoven $\mathrm{R}$, et al. Less radiographic progression with adalimumab plus methotrexate versus methotrexate monotherapy across the spectrum of clinical response in early rheumatoid arthritis. J Rheumatol 2009;36:1429-41.

16. Smolen JS, Han C, van der Heijde DM, et al. Radiographic changes in rheumatoid arthritis patients attaining different disease activity states with methotrexate monotherapy and infl iximab plus methotrexate: the impacts of remission and tumour necrosis factor blockade. Ann Rheum Dis 2009;68:823-7.

17. Singh JA, Wells GA, Christensen R, et al. Adverse effects of biologics: a network meta-analysis and Cochrane overview. Cochrane Database Syst Rev 2011:CD008794.

18. Burmester GR, Mease P, Dijkmans BA, et al. Adalimumab safety and mortality rates from global clinical trials of six immune-mediated infl ammatory diseases. Ann Rheum Dis 2009;68:1863-9.

19. Galloway JB, Hyrich $\mathrm{KL}$, Mercer LK, et al. Anti-TNF therapy is associated with an increased risk of serious infections in patients with rheumatoid arthritis especially in the fi rst 6 months of treatment: updated results from the British Society for Rheumatology Biologics Register with special emphasis on risks in the elderly. Rheumatology (Oxford) 2011;50:124-31.

20. Curtis JR, Xie F, Muntner PM, et al. Does association between anti-TNF biologics and serious infections in rheumatoid arthritis patients vary by comorbidity burden? (abstract) Arthritis Rheum 2011:63(Suppl 10):P426.

21. Askling J, Fored CM, Brandt $L$, et al. Time-dependent increase in risk of hospitalisation with infection among Swedish RA patients treated with TNF antagonists. Ann Rheum Dis 2007;66:1339-44.

22. Busquets N, Tomero E, Descalzo MÁ, et al. Age at treatment predicts reason for discontinuation of TNF antagonists: data from the BIOBADASER 2.0 registry. Rheumatology (Oxford) 2011:50:1999-2004. 